\title{
Peripheral Hypertension-How to Normalize Pressure and the Pulse with no Medications in Several Days with the Clavitherapy Method?
}

\author{
Ferdynand Barbasiewicz* \\ Author of Clavitherapy, University of Warsaw, Poland
}

Received: September 10, 2017; Published: September 20, 2017

*Corresponding author: Ferdynand Barbasiewicz, Author of Clavitherapy, University of Warsaw, Warsaw, Poland, Europe, Email: centrum@klawiterapia.com

\section{Opinion}

The molecular method of biocybernetic clavitherapy has been liquidating hypertension remarkably effectively with no medications since the $40 \mathrm{~s}$. With child's ease in minutes by simple procedures you can yourselves with no medications effectively and permanently liquidate drug-resistant hypertension. But it is necessary to relieve cortical tensions early by stimulation gently pressing with two toothpicks between the brows over 3 seconds at the base of the nose and with the other hand with two toothpicks at the back of the head right under the occipital bone on the meridian line for relieving central tensions. The procedure must be repeated several times until pain and burning recede. Next stimulate the projection of the $5^{\text {th }}$ cranial nerve right under the dura mater of the occipital bone halfway through the distance between the median line and the mammary gland. After a few 3 -second presses, receding of pain and burning also check in the middle of the fontanelle bone that there is no projection of pain and burning. There is the vascular and motor centre of the blood supply of the brain there. The projection of the zone of pressure regulation in the brain PaM-1 will be indicated by the doctor knowing acupuncture.

How to perform stimulation in a decrease in peripheral pressure? Take between the fingers two well-known sharp toothpicks and de-stress at the very bottom the $4^{\text {th }}$ chamber of the brainstem-at the very bottom there is the vascular and motor centre of peripheral pressure. Where to stimulate, press for a few seconds with the toothpicks? The bottom part of the external ear precisely assigns the projection of the sympathetic nervous system in the Atlas of Clavitherapy, a biologically active place between the upper part of the angle of the mandible and the mammary, clavicular and sternum muscle, of course on both sides. Deep at the very bottom of the brainstem there is a vascular and motor centre. After several-second presses with the toothpicks on both sides, when the pain and burning pass, the pressure will return to normal. Please examine the pressure condition on both arms.

The experiment is best to be performed at the presence of a primary care physician. If you are on medication and have a pressure within 130-150/90-100 with a tendency for tachycardia, after a few minutes of stimulation, it will amount to 100-110/70-80 and there will be no tachycardia, the pulse amounting to 65-80. And if the pulse keeps being at the bottom tachycardia, non-invasively press with two toothpicks with the sharp side on the forearm from the inner hand side, from the first fold a few times for 3 seconds each $4-6 \mathrm{~cm}$ above the median nerve towards the cubital joint. In acupuncture it is the 6 th point on the pericardium meridian. Within the space of $2 \mathrm{~cm}$ you have to seek the painful spot on a median basis between the radial bone and the cubital one.

As you remove the pain and burning, there will be no tachycardia and simultaneously you will activate stressed parathyroid glands and they will better start dosing calcium ions to the peripheral blood, which will improve the absorption of calcium from the nourishment you consume in the digestive system. After lowering the pressure the doctor should put aside medications for lowering pressure. Clavitherapy procedures must keep be applying over a week daily for testing and later running a check occasionally after more severe stressful and emotional experiences. It is essential to check geopathic radiations in the home, which should be performed by an experienced diviner or dowser who digs wells for procuring water and buy a good re-radioator, screen. 


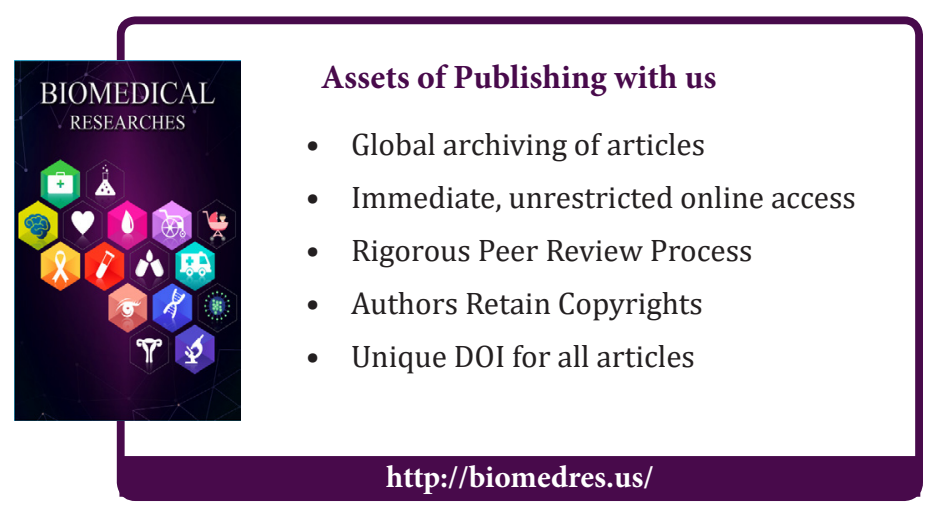

